\title{
CLINICAL SPOTLIGHT
}

\section{Selenium supplementation for Hashimoto's thyroiditis}

\section{Esther J van Zuuren}

Department of Dermatology B1-Q, Leiden University Medical Centre, PO box 96002300 RC Leiden, the Netherlands, Email: e.j.van_zuuren@lumc.nl

Hashimoto's thyroiditis, or chronic lymphocytic thyroiditis, is a common auto-immune disorder. Hashimoto's thyroiditis tends to run in families and affects women and men of all ages, although it is most often seen in middle-aged women. ${ }^{1,2}$ Its prevalence is influenced by ethnicity, environmental factors such as iodine and selenium status, age and gender. ${ }^{1,2}$ The most common presenting symptoms may include anxiety, negative mood, depression, dry skin, cold intolerance, puffy eyes, muscle cramps and fatigue, deep voice, constipation, slow thinking and poor memory. ${ }^{3,4}$ Specific serum auto-antibodies such as anti-thyroid peroxidase antibodies and anti-thyroglobulin antibodies are characteristic of Hashimoto's thyroiditis; serum thyroxine (T4) may be normal or low, and thyroid-stimulating hormone (TSH) concentrations may be normal or high. ${ }^{5}$ Histopathologic examination merely shows diffuse lymphocytic infiltration and formation of germinal centres, although fibrosis can also be detected., Clinical manifestations of the disease are defined primarily by low levels of thyroid hormones; therefore patients are by hormone replacement therapy, which usually consists of levothyroxine (LT4). ${ }^{7}$

Selenium is an essential trace element that is required in small amounts for correct functioning of the immune system. The recommended daily intake for adults is $55 \mu \mathrm{g} / \mathrm{day} .{ }^{8}$ It is obtained from natural selenium-rich sources such as Brazil nuts, organ meat, muscle meat, cereals, shellfish and fish. ${ }^{9}$ The selenium content of food depends on local soil conditions, which can vary depending on geographical and geological factors. ${ }^{9}$ The serum selenium concentration is believed to be in the 70 to $130 \mathrm{ng} / \mathrm{mL}$ range. ${ }^{10}$ Selenomethionine and sodium selenite are the two most common oral forms of selenium supplementation that are available in variable dosages (100 and $200 \mu \mathrm{g} /$ day) and are usually taken for Hashimoto's thyroiditis. ${ }^{11}$ Several studies have suggested that selenium supplementation in patients with Hashimoto's thyroiditis reduces antibodies levels, results in a decreased dosage of LT4 and may provide other beneficial effects (e.g., on mood and health-related quality of life). ${ }^{12}$ We conducted a systematic review to assess the effects of selenium supplementation for Hashimoto's thyroiditis. ${ }^{13}$

We included randomised controlled trials in adults diagnosed with Hashimoto's thyroiditis. Selenium $100 \mu \mathrm{g}$ or $200 \mu \mathrm{g}$ (sodium selenite or selenomethionine) only or combined with titrated LT4 (to maintain basal TSH within normal range) was compared with: no selenium or no selenium plus titrated LT4 respectively; placebo tablets or placebo tablets plus titrated LT4 respectively.

Four studies at unclear to high risk of bias comprising 463 participants were included. The mean study duration was 7.5 months (range 3 to 18 months). None of the studies addressed our key primary outcome-'health-related quality of life'. 
Two of our secondary outcomes-'change from baseline in levothyroxine (i.e., thyroid hormone) replacement dosage at end of the study' and 'economic costs' - were not assessed either. One study at high risk of bias showed a statistically significant improvement in subjective well-being with sodium selenite $200 \mu \mathrm{g}$ plus levothyroxine compared with placebo plus levothyroxine (14/18 compared with 3/18, respectively). Selenomethionine $200 \mu \mathrm{g}$ reduced the serum levels of anti-thyroid peroxidase antibodies in three studies, and although the changes from baseline were statistically significant, their clinical relevance is unclear. Adverse events were reported in two studies, and selenium supplementation did not lead to more adverse events than were seen with placebo. Results of these four studies show that evidence to support or refute the efficacy of selenium supplementation in people with Hashimoto's thyroiditis is incomplete. This does not at present allow confident decision making about the use of selenium supplementation for Hashimoto's thyroiditis.

\section{References}

1. Fink H, Hintze G. [Autoimmune thyroiditis (Hashimoto's thyroiditis): current diagnostics and therapy.]. Med Klin (Munich). 2010;105(7):485-93.

2. Stathatos N, Daniels GH. Autoimmune thyroid disease. Curr Opin Rheumatol. 2012;24(1):70-5.

3. Canaris GJ, Manowitz NR, Mayor G, et al. The Colorado thyroid disease prevalence study. Arch Intern Med. 2000;160(4):526-34.

4. Carta MG, Loviselli A, Hardoy MC, et al. The link between thyroid autoimmunity (antithyroid peroxidase autoantibodies) with anxiety and mood disorders in the community: a field of interest for public health in the future. BMC Psychiatry. 2004;4:25.
5. Li Y, Nishihara E, Kakudo K. Hashimoto's thyroiditis: old concepts and new insights. Curr Opin Rheumatol. 2011;23(1):102-7.

6. Stuart A. The changing scene in Hashimoto's disease: a review. Med Hypotheses. 2011;77(3):424-6.

7. Özen S, Berk O, Şimşek DG, et al. Clinical course of Hashimoto's thyroiditis and effects of levothyroxine therapy on the clinical course of the disease in children and adolescents. J Clin Res Pediatr Endocrinol. 2011;3(4):192-7.

8. Hu Y, McIntosh GH, Young GP. Selenium-rich foods: a promising approach to colorectal cancer prevention. Curr Pharm Biotechnol. 2012;13(1):165-72.

9. Rayman MP. Food-chain selenium and human health: emphasis on intake. $\mathrm{Br} \mathrm{J}$ Nutr. 2008;100(2):254-68.

10. Bleys J, Navas-Acien A, Guallar E. Serum selenium levels and all-cause, cancer, and cardiovascular mortality among US adults. Arch Intern Med. 2008;168(4):404-10.

11. Toulis KA, Anastasilakis AD, Tzellos TG, et al. Selenium supplementation in the treatment of Hashimoto's thyroiditis: a systematic review and a metaanalysis. Thyroid. 2010;20(10):1163-73.

12. Ott J, Promberger R, Kober F, et al. Hashimoto's thyroiditis affects symptom load and quality of life unrelated to hypothyroidism: a prospective case-control study in women undergoing thyroidectomy for benign goiter. Thyroid. 2011;21(2):161-7.

13. van Zuuren E, Albusta AY, Fedorowicz Z, et al. Selenium supplementation for Hashimoto's thyroiditis. Cochrane Database Syst Rev. 2013;6:CD010223. 